\title{
CHALlENGeS OF TREATING YOUNG WOMEN WITH CANCER IN THE ERA OF NEW ONCOLOGIC TREATMENTS
}

\author{
María T. Bourlon ${ }^{1}$, Regina Barragan-Carrillo ${ }^{1}$, Fernanda Mesa-Chavez ${ }^{2,3}$, And \\ CYNTHIA VILLARREAL-GARZA $2,3, *$ \\ ${ }^{1}$ Hematology-Oncology Department, Instituto Nacional de Ciencias Médicas y Nutrición Salvador Zubirán, Mexico City, \\ Mexico; ${ }^{2}$ Breast Cancer Center, Hospital Zambrano Hellion TecSalud, Tecnologico de Monterrey, San Pedro Garza \\ García, NL, Mexico; ${ }^{3}$ Joven \& Fuerte: Programa para la Atención e Investigación de Mujeres Jóvenes con Cáncer de \\ Mama, Mexico City, Mexico
}

\begin{abstract}
Young women with cancer comprise a special population of patients who experience cancer and oncologic care in a unique way. Recent progress in diagnostic and therapeutic approaches has transformed the landscape of clinical oncology practice. This perspective addresses novel therapies, and some of the main challenges that oncologists face when providing care for young patients in the era of next-generation sequencing and tissue-agnostic approaches through the use of targeted therapies for diverse malignancies. (REV INVEST CLIN. 2021;73(5):302-5)
\end{abstract}

Key words: Breast cancer. Thyroid cancer,. Cervical cancer. Young women and cancer.

In recent years, the incidence and prevalence of cancer in young adults (individuals aged 20-39 years) have increased progressively. To date, breast, thyroid, and cervical carcinomas are by far the most commonly diagnosed malignancies in this age group, affecting females either exclusively or predominantly ${ }^{1}$. Novel advances in oncology and related fields have improved physicians' ability to provide integral care for young women with cancer. However, in this era, oncologists are still challenged to aid this young group in solving health-care issues while also addressing the possible repercussions that cancer and oncologic treatment may have on diverse areas of their lives.
Recent progress in different fields of oncology has enabled more precise diagnoses and increased the availability of new targeted treatment options for cancer patients. These novel technologies and therapies have been approved independently of patients' age. Nevertheless, young women especially benefit from these advances for particular reasons, including the high representation of females in the young adult cancer population and their improved life expectancy, which allows them to continue fulfilling their active social roles. However, these scientific breakthroughs can also lead to unique challenges among young patients, mainly emerging toxicities, well-known oncofertility issues
*Corresponding author:

Cynthia Villarreal-Garza

E-mail: cynthia.villarreal@tecsalud.mx

cynthiavg@gmail.com
Received for publication: 04-06-2021

Approved for publication: 19-06-2021

DOI: $10.24875 / R I C .21000312$

0034-8376 / (c) 2021 Revista de Investigación Clínica. Published by Permanyer. This is an open access article under the CC BY-NC-ND license (http://creativecommons.org/licenses/by-nc-nd/4.0/). 
associated with cancer treatment, survivorship aspects, and access to personalized medicine.

In the latest years, patient care has been revolutionized in view of the commercial availability of nextgeneration sequencing. This technology has enhanced the identification of actionable mutations in daily practice and the subsequent prescription of tissueagnostic therapy. Current guidelines for solid tumors recommend the use of genomic testing in advanced progressive disease to identify potential treatment targets, such as BRCA1/2, BRAF V600E, neurotrophic tyrosine receptor kinase (NTRK) and RET fusions and mutations, $\mathrm{PD}-(\mathrm{L}) 1$ expression, DNA mismatch repairs, microsatellite instability, and mutational burden. In the case of breast cancer, the most frequent germline mutations in BRCA1/2 are associated with DNA homologous recombination repair defects. Thus, these patients are potential candidates for treatment with poly-adenosine-diphosphate-ribose polymerase (PARP) inhibitors, as BRCA1/2-deficient cells are highly sensitive to accumulation of toxic doublestrand breaks, genomic instability, and synthetic lethality caused by PARP inhibition ${ }^{2}$. Moreover, the olaparib (OlimpiAD) and talazoparib (EMBRACA) trials have shown significant benefit on progression-free survival benefit with the use of these agents in patients with advanced HER2-negative disease ${ }^{2,3}$. Furthermore, the recently presented results from the OlympiA trial, a novel Phase III trial of olaparib as adjuvant therapy in patients with high-risk HER2negative breast cancer and germline BRCA1/2 mutations represent the first step into personalized treatment in a curative-intent setting ${ }^{4}$.

As for thyroid cancer, even though patients usually have a good prognosis, approximately $50 \%$ of those with metastatic disease can become refractory to first-line treatment with radioactive iodine and thyroid suppressive therapy. In these cases, novel targeted approaches have yielded favorable results. The phase I/II LIBRETTO-001 and ARROW trials demonstrated the significant benefit of RET directed therapies, selpercatinib and pralsetinib, in the context of RET-altered advanced solid cancers in terms of overall response rates and safety profile ${ }^{5,6}$. The main drawback to these approaches is the limited number of tumors that carry an actionable mutation in this context, which in these trials were mainly restricted to medullary thyroid and lung cancer. In the particular case of anaplastic thyroid carcinoma, a neoplasia known for its obscure prognosis, the development of targeted therapies represents one of the few advances that have translated into improved outcomes. In patients with BRAF V600E mutations, dabrafenib plus trametinib have demonstrated important partial response rates, with lasting response durations ${ }^{7}$.

The NTRK fusion-positive tumors can also be subjected to targeted therapy given that selective inhibitors of these proteins have shown robust activity with a prolonged overall response duration ${ }^{8,9}$. Two of these small molecules, larotrectinib and entrectinib, are now approved for the treatment of NTRK fusionpositive solid tumors refractory to prior treatment lines, regardless of the site of disease origin.

Besides targeted therapies, immunotherapy represents one of the newest treatment options for patients with different types of cancer. Through wholegenome sequencing, those who could benefit from such therapies can be readily identified. The Phase II KEYNOTE 158 trial described the benefit of the PD-1 inhibitor pembrolizumab in the management of heavily pre-treated patients with advanced solid tumors and high tumor mutational burden. Even though median progression-free and overall survival did not significantly improve, the high tumor mutational burden subgroup could render a robust tumor response ${ }^{10}$. On a similar note, previously treated advanced microsatellite instability - high or mismatch repair - deficient advanced tumors derive benefit on response rate and lasting response duration ${ }^{11}$.

Accordingly, nowadays, histologic cancer diagnosis is not enough, and oncologists must strive for geneticbased tumor classification to identify patients who could benefit from these promising directed therapies. In addition, it is fundamental that physicians continue contributing to expanding research on these novel potential targets and treatment options.

On a related matter, the increasing use of new therapies is transforming the landscape of clinical oncology practice due to the emergence of novel toxicity profiles that differ from those of classic cancer treatment. Most adverse effects related to treatment modalities such as immunotherapy or targeted therapy are mild and reversible if addressed promptly ${ }^{12,13}$. However, certain toxicities, including adrenal 
insufficiency, hepatitis, myocarditis, encephalitis, pneumonitis, Stevens-Johnson syndrome/toxic epidermal necrolysis, and drug reaction with eosinophilia and systemic symptoms (DRESS), could be life threatening and highlight the importance of observant clinical suspicion ${ }^{12,13}$. Moreover, others such as endocrinopathies and rheumatologic adverse effects might cause permanent organ dysfunction and require chronic or lifelong treatment ${ }^{12,13}$. Besides, since these agents can be prescribed as combination therapy in certain cases, proficiency on their possible toxicities is paramount to recognize properly the effects that need focused management and should not be ascribed to other treatments such as chemotherapy ${ }^{12}$. In addition, while modern immunotherapy and targeted therapies are currently used mostly in advanced settings, they are progressively becoming approved options for earlier cancer stages ${ }^{12,13}$. Thus, oncologists are increasingly facing toxicities that were previously rarely observed in oncologic patients and that require timely identification and specific management ${ }^{13}$. Furthermore, with broader approval of these drugs for early malignancies, a greater volume of patients will be exposed to their possible adverse effects, resulting in a larger toxicity burden.

In young patients, toxicities associated with new therapies might be especially challenging considering the rising prevalence of diverse types of cancer ${ }^{14}$. Particularly, serious and long-term adverse effects could have an important and prolonged negative impact on these patients' overall health and quality of life due to their early age and continuously longer survival times $^{12}$. Hence, it is imperative for oncologists to become familiar with these therapies' unique toxicity profiles. Some of the most relevant oncology associations such as ASCO and NCCN have recommended updated oncologists' knowledge and training, as well as vigilant monitoring and multidisciplinary toxicity team management for integral patient care whenever these therapies are used ${ }^{15,16}$. In addition, patient education about the serious adverse effects they might experience could facilitate toxicity identification and management ${ }^{15,16}$. These strategies might prove crucial to improve patient outcomes and well-being. Also related to oncologic treatment toxicities, the wellknown risk of infertility remains one of the main challenges encountered when providing care for young women with cancer and is a special concern that oncologists should address. In contrast to past decades, oncofertility is now an established field of medicine and a health-care quality standard for young adults undergoing cancer therapy ${ }^{17}$. Several options are currently available for this age group, as fertility preservation strategies have evolved from surgical procedures aimed to diminish radiation gonadotoxicity, to oocyte/embryo cryopreservation, ovarian tissue freezing, temporary ovarian suppression with GnRH analogs during chemotherapy, and even uterine transplantation ${ }^{18,19}$. Furthermore, novel fertility-protective treatments which could be administered before or during gonadotoxic treatment are under development to prevent permanent damage to ovarian tissue ${ }^{18,20,21}$.

Nonetheless, fertility preservation strategies - except for uterine transplantation - depend on the availability of a functional female reproductive system. This is particularly relevant for many patients with gynecological cancers, in which standard-of-care hystero-oophorectomy leads to permanent fertility loss. To overcome this limitation, studies on biomaterials have been actively conducted ${ }^{18}$. Researchers have attempted to develop long-lasting transplantable reproductive tissues through the use of biomaterials arising from different cell sources (such as stem cells or endometrial cells) and paracrine factors ${ }^{18,20,22}$. Therefore, in coming years, updated knowledge and timely referral will be essential for oncologists to help young women with cancer navigate through the diverse fertility preservation options that are becoming available.

Another aspect that continues to represent a challenge for both patients and oncologists is related to the various survivorship issues that young women encounter in a unique way. This age group is particularly vulnerable to cancer and treatment repercussions due to the family, academic, and professional roles that they usually strive to balance during this active time of their lives. Notably, fertility, sexual, body image, emotional, cognitive, and physical adverse effects may importantly compromise the quality of life of young survivors in the short and long terms. Oncologists can play a major role in diminishing these sequelae by addressing young patients' particular concerns throughout their cancer trajectory and providing multidisciplinary, integral care ${ }^{23}$. Moreover, considering young patients' prolonged expected survival times, offering sustained support during the 
survivorship period is fundamental for them to successfully cope with cancer aftermath and oncologic treatment toxicities ${ }^{24}$.

Access to health care is a pressing matter in the era of new oncologic treatment. Both patients and physicians are challenged by the lacking availability of novel diagnostic strategies and treatment options, as well as by the financial drawbacks that prevent cancer patients from receiving cutting-edge care. Continued endeavors to increase access to new therapies that impact on patients' prognosis, as well as interventions to alleviate treatment toxicities, are of paramount importance for these advances to become a reality for most patients.

\section{REFERENCES}

1. Vaccarella S, Ginsburg O, Bray F. Gender inequalities in cancer among young adults. Lancet Oncol. 2021;22:166-7.

2. Litton JK, Hurvitz SA, Mina LA, Rugo HS, Lee KH, Gonçalves A, et al. Talazoparib versus chemotherapy in patients with germline BRCA1/2-mutated HER2-negative advanced breast cancer: final overall survival results from the EMBRACA trial. Ann Oncol. 2020;31:1526-35.

3. Robson M, Im S-A, Senkus E, Xu B, Domchek SM, Masuda N, et al. Olaparib for metastatic breast cancer in patients with a germline BRCA mutation. N Engl J Med. 2017;377:523-33.

4. Tutt A, Garber JE, Kaufman B, Viale G, Fumagalli D, Rastogi P, et al. OlympiA: A phase III, multicenter, randomized, placebocontrolled trial of adjuvant olaparib after (neo)adjuvant chemotherapy in patients with germline BRCA1/2 mutations and highrisk HER2-negative early breast cancer. J Clin Oncol 2021; 39(suppl 15, Abstr LBA1).

5. Hu M, Subbiah V, Wirth LJ, Schuler M, Mansfield AS, Brose MS, et al. Results from the registrational phase I/II ARROW trial of pralsetinib (BLU-667) in patients (pts) with advanced RET mutation-positive medullary thyroid cancer (RET+ MTC). Ann Oncol. 2020;31:S1084.

6. Wirth LJ, Sherman E, Robinson B, Solomon B, Kang H, Lorch J, et al. Efficacy of selpercatinib in RET-altered thyroid cancers . N Engl J Med. 2020;383:825-35

7. Subbiah V, Cabanillas ME, Kreitman RJ, Wainberg ZA, Cho JY, Keam $B$, et al. Dabrafenib and trametinib treatment in patients with locally advanced or metastatic BRAF V600-mutant anaplastic thyroid cancer. J Clin Oncol. 2018;36:7-13.
8. Hong DS, DuBois SG, Kummar S, Farago AF, Albert CM, Rohrberg $\mathrm{KS}$, et al. Larotrectinib in patients with TRK fusion-positive solid tumours: a pooled analysis of three phase $1 / 2$ clinical trials. Lancet Oncol. 2020;21:531-40.

9. Doebele RC, Drilon A, Paz-Ares L, Siena S, Shaw AT, Farago AF et al. Entrectinib in patients with advanced or metastatic NTRK fusion-positive solid tumours: integrated analysis of three phase 1-2 trials. Lancet Oncol. 2020;21:271-82.

10. Marabelle A, Le DT, Ascierto PA, Di Giacomo AM, de JesusAcosta A, Delord JP, et al. Efficacy of pembrolizumab in patients with noncolorectal high microsatellite instability/mismatch repair-deficient cancer: results from the phase II KEYNOTE-158 study. J Clin Oncol. 2020;38:1-10.

11. Marcus L, Lemery SJ, Keegan P, Pazdur R. FDA approval summary: pembrolizumab for the treatment of microsatellite instability-high solid tumors. Clin Cancer Res. 2019;25:3753-8.

12. Johnson DB, Jakubovic BD, Sibaud V, Sise ME. Balancing cancer immunotherapy efficacy and toxicity. J Allergy Clin Immunol Pract. 2020;8:2898-906.

13. Kroschinsky F, Stölzel F, von Bonin S, Beutel G, Kochanek M, Kiehl $M$, et al. New drugs, new toxicities: severe side effects of modern targeted and immunotherapy of cancer and their management. Crit Care. 2017;21:89.

14. Siegel RL, Miller KD, Jemal A. Cancer statistics, 2020. CA Cancer J Clin. 2020;70:7-30.

15. Cousin S, Seneschal J, Italiano A. Toxicity profiles of immunotherapy. Pharmacol Ther. 2018;181:91-100.

16. Naidoo J, Zhang J, Lipson EJ, Forde PM, Suresh K, Moseley KF, et al. A multidisciplinary toxicity team for cancer immunotherapy-related adverse events. JNCCN J Natl Compr Cancer Netw. 2019;17:712-20.

17. Bourlon MT, Anazodo A, Woodruff TK, Segelov E. Oncofertility as a universal right and a global oncology priority. JCO Glob Oncol. 2020;2018:314-6.

18. Somigliana E, Mangili G, Martinelli F, Noli S, Filippi F, Bergamini A, et al. Fertility preservation in women with cervical cancer. Crit Rev Oncol Hematol. 2020;154:103092.

19. Ejzenberg D, Andraus W, Mendes LR, Ducatti L, Song A, Tanigawa $\mathrm{R}$, et al. Livebirth after uterus transplantation from a deceased donor in a recipient with uterine infertility. Lancet. 2018;392:2697-704.

20. Hao X, Anastácio A, Liu K, Rodriguez-Wallberg KA. Ovarian follicle depletion induced by chemotherapy and the investigational stages of potential fertility-protective treatments-A review. Int J Mol Sci. 2019;20:4720.

21. Morgan S, Lopes F, Gourley C, Anderson RA, Spears N. Cisplatin and doxorubicin induce distinct mechanisms of ovarian follicle loss; imatinib provides selective protection only against cisplatin. PLoS One. 2013;8:e70117.

22. Christianson MS, Oktay K. Advances in fertility-preservation surgery: navigating new frontiers. Fertil Steril. 2019;112: 438-45.

23. Partridge $\mathrm{AH}$. Cancer survivorship and the young breast cancer patient: Addressing the important issues. Oncologist. 2013; 18:e19-20.

24. Nardin S, Mora E, Varughese FM, D'Avanzo F, Vachanaram AR, Rossi $V$, et al. Breast cancer survivorship, quality of life, and late toxicities. Front Oncol. 2020;10:864. 